\title{
Alinhando o modelo, o método de implementação e a prática de gestão do conhecimento (GC): caso do Repositório do Conhecimento do Instituto de Pesquisa Econômica Aplicada (RClpea)
}

Fábio Ferreira Batista e Veruska da Silva Costa

\section{Introdução}

Entre as decisões mais importantes para o êxito da implementação da gestão do conhecimento (GC) nas organizações, encontram-se as escolhas do modelo, do método de implementação e das práticas mais adequadas para alcançar os resultados esperados com a iniciativa.

Organizações públicas brasileiras vêm adotando modelos distintos no momento de implementar GC (Barbosa, 2011 e Alvarenga Neto e Vieira, 2011). O objetivo deste artigo é analisar como o Instituto de Pesquisa Econômica Aplicada (Ipea) implementou de forma alinhada um modelo, um método e uma prática de gestão do conhecimento (GC). O referencial teórico utilizado é o Modelo de Gestão do Conhecimento para a Administração Pública Brasileira, construído por um dos autores deste trabalho após a realização de uma revisão sistemática da literatura sobre modelos de GC na administração pública (BATISTA, 2012).

Além dessa introdução, este artigo é composto de três seções. Na primeira, "Modelo teórico e conceitual de gestão do conhecimento (GC)", definimos 
conceitos, apresentamos o referencial teórico e detalhamos o Modelo de Gestão do Conhecimento para a Administração Pública Brasileira - proposto e adotado pelo Instituto de Pesquisa Econômica Aplicada (Ipea). Em seguida, descrevemos o roteiro para a elaboração do plano de GC utilizado pela instituição.

Na segunda seção, "Implementação da gestão do conhecimento no Ipea", apresentamos o perfil organizacional do Ipea e analisamos como a fundação elaborou e implementou o projeto RCIpea com base no modelo e no roteiro escolhidos.

Finalmente, na terceira e última seção, "Considerações finais", destacamos os resultados da implementação da gestão do conhecimento para a instituição.

\section{Modelo teórico e conceitual de gestão do conhecimento (GC)}

Para uma melhor compreensão, apresentamos, a seguir, algumas definições de conceitos importantes adotadas neste trabalho e extraídas da literatura sobre GC:

i) Gestão do conhecimento na admi nistração pública brasileira:

“(...) um método integrado de criar, compartilhar e aplicar o conhecimento para aumentar a eficiência; melhorar a qualidade e a efetividade social; e contribuir para a legalidade, impessoalidade, moralidade e publicidade na administração pública e para o desenvolvimento brasileiro." (BАTISTA, 2012, p. 49)

ii) Conhecimento:

“(...) uma mistura fluida de experiência condensada, valores, informação contextual e insight experimentado, a qual proporciona uma infraestrutura para avaliação e incorporação de novas experiências e informações. Ele tem origem e é aplicado na mente dos conhecedores. Nas organizações, ele costuma estar embutido não só em documentos ou repositórios, mas também nas rotinas, processos, práticas e normas organizacionais." (DAVENPORT e Prusak, 1998, p. 5)

iii) Conhecimento tácito:

“(...) é altamente pessoal e difícil de formalizar, sendo difícil de ser comunicado ou compartilhado com outros. Insights subjetivos, intuições e dicas integram esta categoria de conhecimento. Além disso, conhecimento tácito é profundamente enraizado na ação e experiência do indivíduo, assim como nos ideais, valores ou emoções abraçados por ele ou ela." (Nonaka e TAkeuchi, 1995, p. 8)

iv) Conhecimento explícito:

“(...) pode ser expresso em palavras e números, e facilmente comunicado e compartilhado na forma de dados, fórmulas científicas, procedimentos codificados ou princípios universais (...) pode ser facilmente 'processado' por um computador, transmitido eletronicamente ou armazenado em banco de dados." (NonAkA e TAKeuCHI, 1995, pp. 8-9)

v) Repositório do conhecimento:

"Repositórios do conhecimento são normalmente tipos de intranets ou 
portais que servem para preservar, gerenciar e alavancar a memória organizacional (...) Há vários tipos diferentes de repositórios do conhecimento utilizado hoje (...) eles podem ser classificados de maneiras diferentes. Em geral, um repositório de conhecimento conterá mais do que documentos (sistema de gestão de documentos), dados (banco de dados), ou registros (sistema de gestão de registros). Um repositório do conhecimento conterá conhecimento valioso que é uma mistura de conhecimento tácito e explícito, baseado nas experiências únicas dos indivíduos que são ou foram parte daquela companhia, assim como o Knowhow que tem sido tentado, testado e demonstrado como bem sucedido em situações de trabalho.” (DALKIR, 2011, pp. 213-214)

Ao realizar uma revisão sistemática da literatura sobre modelos de GC na obra "Modelo de GC para a Administração Pública (AP) Brasileira", Batista conclui que: i) há poucos estudos que propõem modelos específicos para a AP (SALAVATI, Shafei e Shaghayegh, 2010 e Monaviarian e Kasaei, 2007); ii) é muito frequente encontrar estudos de caso nos quais os autores utilizam modelos já existentes; iii) são escassos os trabalhos propondo modelos de GC holísticos e específicos para a AP; e iv) até os poucos modelos de GC para a AP não apresentam contribuições relevantes para a implementação da GC na AP brasileira (BAtista, 2012).

À luz da revisão de literatura e baseado em modelos, instrumentos de avaliação e roteiros de implementação da GC utilizados na AP, Batista constrói e propõe o Modelo de GC para a AP Brasileira (Salavati, Shafei e Shaghayegh, 2010; Fonseca, 2006; Instrumento Para A Avaliação Da Gestão Pública, Ciclo 2010; Apo, 2009; OecD, 2003; The Know Network, 2011; European Km Forum, 2001; Heisig, 2009, entre outros).

As seguintes características desse modelo, entre outras, levaram o Ipea a adotá-lo: i) simplicidade; ii) praticidade;

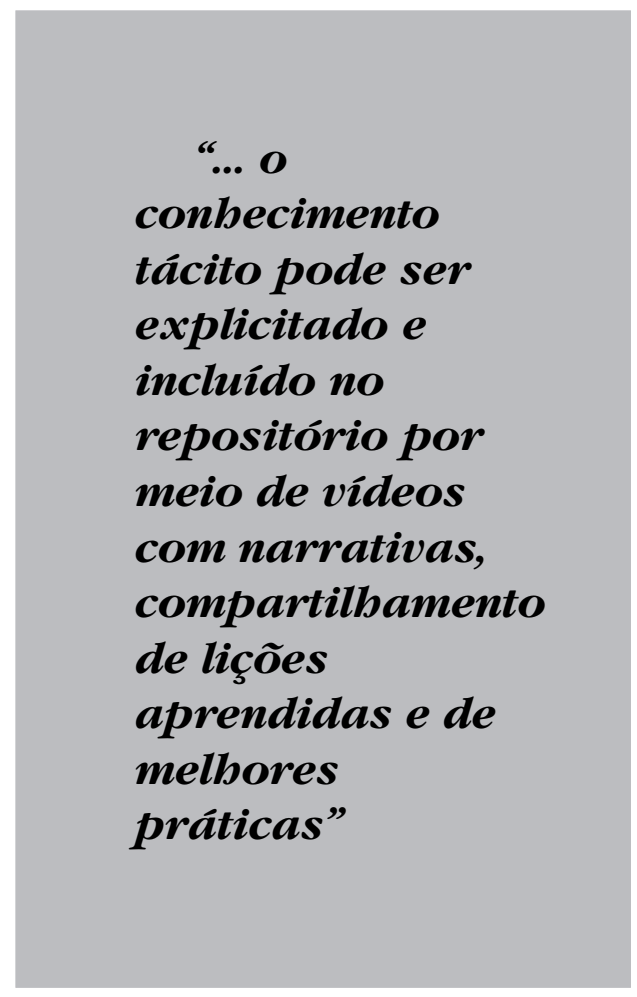

iii) definição clara, objetiva e contextualizada de GC para a AP; iv) sólida fundamentação teórica; v) relevância e utilidade para a AP; vi) contempla fatores críticos de sucesso; e vi) manual de implementação de GC que acompanha a obra (BATista, 2012).

Passamos, a seguir, a descrever esse modelo. 
Modelo de Gestão do Conhecimento para a Administração Pública Brasileira

A Figura 1 mostra os seis componentes do Modelo de Gestão do Conhecimento para a Administração Pública Brasileira proposto e utilizado pelo Ipea: i) direcionadores estratégicos: visão, missão, objetivos estratégicos, estratégias e metas; ii) viabilizadores: liderança, tecnologia, pessoas e processos; iii) ciclo de GC: identificar, criar, armazenar, compartilhar e aplicar; iv) ciclo KDCA; v) resultados de GC; e vi) partes interessadas: cidadão-usuário e sociedade (BAтіsтA, 2012).

O modelo foi construído com base nos componentes identificados na revisão da literatura sobre modelos de GC e nos modelos, instrumentos de avaliação e roteiros de implementação de GC utilizados por organizações públicas (BATISTA, 2012).
Os direcionadores estratégicos, isto é, a visão de futuro, a missão institucional, os objetivos estratégicos e as metas, são o primeiro componente e servem de fundamento para o modelo. É essencial para êxito da iniciativa alinhar a Gestão do Conhecimento (GC) com tais direcionadores. Assim, a GC servirá como instrumento para alcançar os resultados organizacionais (BATISTA, 2012).

Os fatores críticos de sucesso ou viabilizadores da GC constituem o segundo componente do modelo. São eles: i) liderança; ii) tecnologia; iii) pessoas; e iv) processos (BAтіsтA, 2012).

O terceiro componente do Modelo de GC para a Administração Pública Brasileira é o processo de GC. Para mobilizar de maneira sistemática o conhecimento para alcançar os objetivos organizacionais, cinco atividades do processo de GC são necessárias: identificar, criar, armazenar,

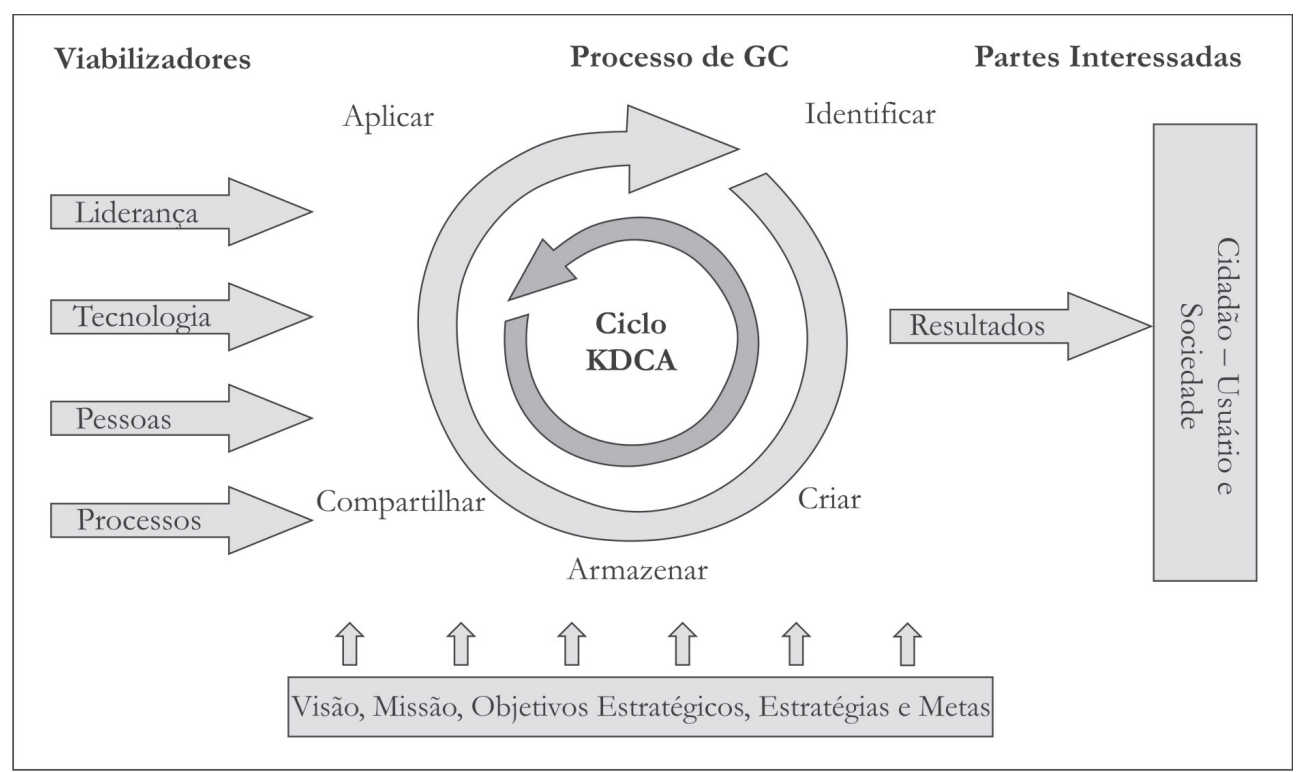

Fonte: BATISTA, 2012.

Figura 1: Modelo de Gestão do Conhecimento para a Administração Pública Brasileira 
compartilhar e aplicar o conhecimento. Essas atividades devem ser colocadas em prática na gestão de processos e de projetos. Para tal, deve-se utilizar o ciclo KDCA (BATISTA, 2012).

O ciclo KDCA é composto de quatro etapas. O objetivo da primeira etapa, Knowledge (conhecimento), é elaborar o plano de GC. Na segunda etapa, Do (executar), a organização deve: 1) educar e capacitar os colaboradores; 2) executar o plano de GC; e 3) coletar dados e informações. Na terceira etapa, Check (verificar), é verificado se a meta de melhoria da qualidade foi alcançada e se o plano de GC foi executado conforme previsto. Finalmente, na etapa $A c t$ (corrigir ou armazenar), caso a meta não tenha sido atingida, a organização corrige eventuais erros nas atividades do processo de GC (identificação, criação, compartilhamento e aplicação do conhecimento). Se a meta foi alcançada, a organização armazena o novo conhecimento por meio da padronização (BATISTA, 2012).

Como se trata de um modelo de GC com foco em resultados, o quinto componente enfoca os resultados da gestão do conhecimento. Há dois tipos de resultados esperados com a implementação da GC: imediatos e finais. Os resultados imediatos são aprendizagem e inovação. Como consequência, há o incremento da capacidade de realização do indivíduo, da equipe, da organização e da sociedade na identificação, criação, armazenamento, compartilhamento e aplicação do conhecimento (BАтіsтA, 2012).

Os resultados finais destacados no modelo são consequência dos resultados imediatos (aprendizagem e inovação; e aumento da capacidade de realização do indivíduo, das equipes, da organização e da sociedade) e consistem em: aumentar a eficiência; melhorar a qualidade e a efetividade social; contribuir para a legalidade, impessoalidade, moralidade e publicidade na administração pública e para o desenvolvimento brasileiro (BATISTA, 2012).

Finalmente, o sexto e último componente do modelo de GC para a administração pública são as partes interessadas, a saber: o cidadão-usuário e a sociedade. É fundamental para a organização pública gerenciar o conhecimento sobre os cidadãosusuários para que ela possa cumprir sua missão e atender as necessidades e expectativas em relação aos serviços prestados. Identificar as necessidades e expectativas da sociedade em geral é uma tarefa relevante na gestão do conhecimento nas organizações públicas. Para a GC na administração pública é importante abordar temas relevantes para a sociedade, tais como: desenvolvimento, responsabilidade pública, transparência, inclusão social, interação e gestão do impacto da atuação da organização na sociedade (BATISTA, 2012).

$\mathrm{Na}$ próxima seção, apresentamos o roteiro para a elaboração do plano de GC utilizado pelo Ipea. Esse roteiro é baseado no Modelo de GC para a Administração Pública Brasileira que acabamos de descrever.

\section{Roteiro para a elaboração do plano de gestão do conheci- mento (GC)}

As seguintes perguntas norteiam as etapas de elaboração do plano de gestão do conhecimento (PGC): i) Onde estamos agora? ii) Onde queremos estar? iii) Como chegaremos lá? iv) Quais são as métricas ou indicadores para avaliar o grau de sucesso? e v) Estamos alcançando nossos objetivos e visão de GC? 
Para responder a pergunta "Onde estamos agora?” (etapa 1), a organização pública deve, em primeiro lugar, realizar a autoavaliação do grau de maturidade em GC (passo 1). A autoavaliação é baseada em sete critérios do instrumento para a avaliação do grau de maturidade em GC, a saber: 1) liderança em GC; 2) processo; 3) pessoas; 4) tecnologia; 5) processo de GC; 6) aprendizagem e inovação; e 7) resultados da GC.

Cada um dos critérios elencados acima é constituído de assertivas - totalizando 42 - que devem ser cuidadosamente analisadas e pontuadas com base em evidências. A autoavaliação permite identificar o grau de maturidade em GC da organização, assim como realizar benchmarking com organizações congêneres. Utilizam-se duas escalas na aplicação do instrumento (BATISTA, 2012).

Após a realização da autoavaliação, a organização identifica seus pontos fortes (PF) e oportunidades de melhoria $\mathrm{OM}$ (passo 2). Aqui é possível identificar o que está bom (PF) e o que precisa ser melhorado (OM) para que ocorra a efetiva institucionalização da GC na organização.

O passo seguinte na elaboração do PGC é identificar as lacunas de conhecimento (passo 3). Uma lacuna do conhecimento é a diferença entre o que a organização sabe e o que ela deveria saber para alcançar seus objetivos estratégicos. É nesse ponto que ocorrerá a intervenção da gestão do conhecimento. No planejamento estratégico procuram-se eliminar as lacunas estratégicas (diferença entre o que a organização faz e o que ela deveria fazer para alcançar seus objetivos estratégicos) por meio da eliminação das lacunas do conhecimento.

Após identificar as lacunas do conhecimento e para responder a pergunta
“Onde queremos estar?” (etapa 2), a organização define a visão de gestão do conhecimento (passo 4), isto é, a situação futura após a implementação da GC em que a lacuna de conhecimento já terá sido eliminada.

Em seguida, para responder a pergunta "Como chegaremos lá?" (etapa 3), a organização deve executar os seguintes passos: i) definição dos objetivos e estratégia de GC (passo 5); ii) elaboração do plano de gestão do conhecimento (PGC) (passo 6); e iii) elaboração do plano de melhoria do grau de maturidade em GC (PMM) (passo 7).

O PGC mostra como a organização eliminará as lacunas do conhecimento, enquanto que o PMM aponta para as ações a serem executadas para trabalhar as oportunidades de melhoria (OM) identificadas na autoavaliação e, consequentemente, para elevar o grau de maturidade em GC na organização.

Em seguida, para responder a pergunta "Quais são as métricas para avaliar o grau de sucesso?” (etapa 4), a organização deverá elaborar os indicadores dos resultados da estratégia (passo 8) para verificar o sucesso na eliminação das lacunas do conhecimento. Para avaliar se está havendo ou não elevação no grau de maturidade em GC, a organização comparará a pontuação obtida no diagnóstico atual com o diagnóstico anterior (passo 9).

Finalmente, para responder a pergunta "Estamos alcançando nossos objetivos e visão de GC” (etapa 5), a organização deve realizar o monitoramento, avaliação e elaboração de relatórios (passo 10).

$\mathrm{Na}$ próxima seção, apresentamos o perfil organizacional do Ipea e descrevemos como a instituição elaborou seu plano de GC com base no roteiro aqui apresentado. 
Implementação da gestão do conhecimento no Ipea

\section{Perfil organizacional}

O Instituto de Pesquisa Econômica Aplicada (Ipea) é uma fundação pública federal vinculada à Secretaria de Assuntos Estratégicos da Presidência da República (SAE/PR). Suas atividades de pesquisa fornecem suporte técnico e institucional às ações governamentais para a formulação e reformulação de políticas públicas e programas de desenvolvimento brasileiros. Os trabalhos do Ipea chegam à sociedade por meio de inúmeras publicações, seminários, assessoramento técnico, cursos e divulgação na imprensa e, mais recentemente, via programa semanal de TV em canal fechado.

\section{Elaboração do plano de gestão do conhecimento (GC) do Ipea}

Seguindo o roteiro para a elaboração do plano de gestão do conhecimento apresentado, o Ipea iniciou a elaboração do seu plano de GC com a realização da autoavaliação. O Ipea obteve uma pontuação de 75,8 de um total de 210 pontos. Com essa pontuação, o Ipea encontra-se ainda na primeira etapa (reação) dos graus de maturidade em GC. As demais etapas são: iniciação (125 pontos), introdução (expansão - 146 pontos), refinamento (188 pontos), e maturidade (210 pontos).

$\mathrm{Na}$ autoavaliação, o Ipea identificou também os pontos fortes (PF) e as oportunidades de melhoria (OM). É importante destacar que, no critério 5.0 Processo de Conbecimento, o Ipea identificou a OM "inexistência de um processo sistematizado de armazenamento, renovação, acesso e utilização do conhecimento produzido na instituição". Essa
OM ajudou a identificar a lacuna do conhecimento em que a intervenção de GC deveria ocorrer.

O Ipea decidiu iniciar a implementação da GC para eliminar a seguinte lacuna estratégica do conhecimento: o conhecimento produzido pelo Ipea não estava representado, organizado e armazenado de forma estruturada e não seguia as recomendações internacionais para a disseminação, acesso e utilização da informação, visando

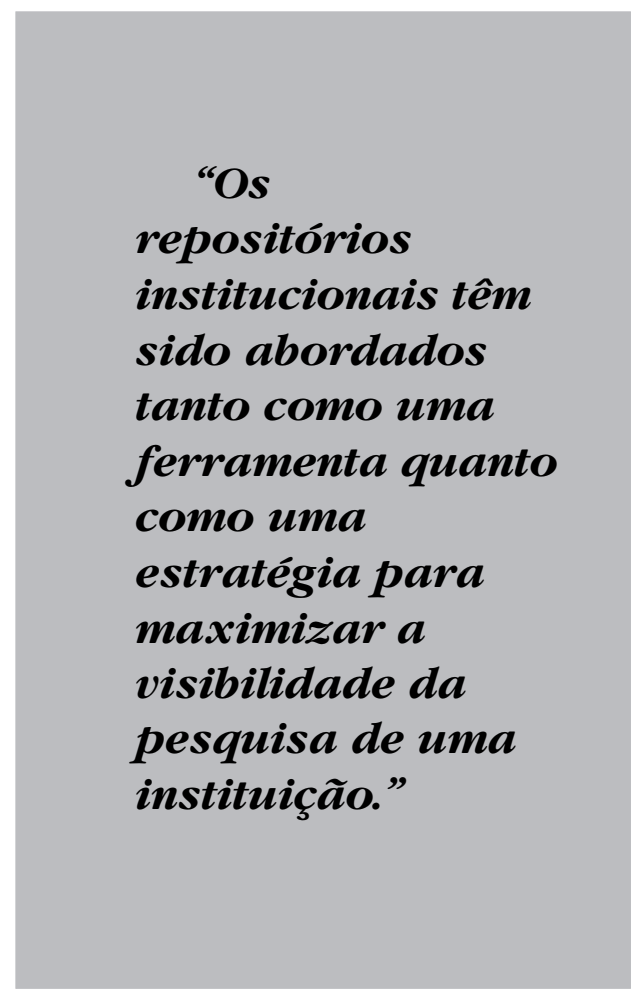

a aumentar a visibilidade da pesquisa e a interoperabilidade em rede.

A situação esperada depois da implementação da GC, isto é, a visão de GC definida pelo Ipea foi: "Conhecimento produzido pelo Ipea representado, organizado e armazenado de forma estruturada, seguindo as recomendações internacionais para a disseminação, acesso 
e utilização da informação, permitindo, assim, sua utilização adequada pelo público interno e externo".

Os principais objetivos definidos pelo Ipea para alcançar a visão de GC foram: i) aumentar a visibilidade e o acesso à pesquisa e a interoperabilidade de toda a produção do Ipea em rede; ii) recuperar os documentos pelo texto completo ou por meio de busca simples e avançada e disponibilizar informações estatísticas de acesso e download; iii) identificar as publicações mais acessadas; iv) mostrar as relações existentes entre as publicações do Ipea, em seus diversos suportes eletrônicos.

Para isso, o instituto definiu a estratégia de implantar processo de representação, organização, armazenamento, renovação, disseminação, acesso e utilização do conhecimento produzido nos processos finalísticos do Ipea.

O Ipea ainda não elaborou o plano de melhoria do grau de maturidade em GC (passo 7). Por isso, passamos agora para o passo seguinte: indicadores de resultados da estratégia.

Para monitorar se a estratégia de GC da organização está sendo bem-sucedida, o Ipea decidiu adotar indicadores relativos aos processos de representação, organização, armazenamento/disseminação, acesso e utilização do conhecimento. Para cada processo foram elaborados indicadores, a saber: i) armazenamento/disseminação, ii) acesso, e iii) utilização.

A autoavaliação deverá se repetir anualmente. Assim, o passo 9 (pontuação na autoavaliação) ocorrerá com essa periodicidade. Já o passo 10 (monitoramento, avaliação e elaboração de relatórios) será executado mensalmente.

Em síntese, o Ipea seguiu o roteiro para a elaboração do plano de gestão do conhecimento apresentado para eliminar a lacuna do conhecimento referente aos processos de representação, organização, disseminação, acesso e utilização do conhecimento produzido.

Na próxima seção, analisamos como o Ipea construiu o protótipo do Repositório do Conhecimento do Ipea (RCIpea).

\section{Repositório do Conhecimento do Ipea (RCIpea)}

A implementação do Repositório do Conhecimento do Ipea (RCIpea) teve por finalidade, portanto, eliminar uma lacuna estratégica do conhecimento referente aos processos de representação, organização, disseminação, acesso e utilização do conhecimento produzido pela instituição.

O RCIpea é uma iniciativa de gestão do conhecimento porque, conforme destaca Dalkir, é um repositório que contém conhecimento valioso que é uma mistura de conhecimento tácito e explícito (DALkIR, 2011). O conhecimento explícito pode ser inserido no RCIpea na forma de livros, artigos, imagens etc. Já o conhecimento tácito pode ser explicitado e incluído no repositório por meio de vídeos com narrativas, compartilhamento de lições aprendidas e de melhores práticas (BATISTA, 2012).

O RCIpea é uma prática de GC associada às atividades de armazenar, compartilhar e aplicar o conhecimento no âmbito do ciclo de GC do Modelo de GC para a AP Brasileira. Além disso, contribui para a melhoria do processo "realização de estudos e pesquisas". Assim sendo, está associado ao viabilizador "processos" do modelo. O RCIpea está vinculado também a todos os passos do roteiro para a elaboração do plano de GC (passo 1: autoavaliação; passo 2: solução para uma oportunidade de melhoria; passo 3: solução 
para lacuna de conhecimento; e assim por diante).

Os repositórios digitais podem ser vistos também como uma prática de GC, ligada à gestão de processos e projetos. Para atingir esse objetivo, o conhecimento no repositório digital deve ser organizado de uma maneira que a aprendizagem seja possível. Dessa forma, há uma preocupação em alimentar o repositório com informações úteis para as equipes de projetos futuros: 1) metodologias de pesquisa empregadas; 2) lições aprendidas com erros e acertos; 3) softwares e base de dados utilizados durante a execução do projeto; 4) questionários e instrumentos de pesquisa; 5) projetos de pesquisa semelhantes, conduzidos por outras instituições de pesquisa etc.

Repositórios institucionais são também repositórios digitais voltados para o armazenamento, preservação e disseminação da produção intelectual de uma instituição (Costa \& LeITE, 2006). Os repositórios institucionais têm sido abordados tanto como uma ferramenta quanto como uma estratégia para maximizar a visibilidade da pesquisa de uma instituição. Essa prática de GC potencializa a troca de conhecimento no seio das comunidades técnicas e científicas e alimenta a preocupação com o acesso à pesquisa e os impactos causados pelo acesso aberto (Open Access) ao conhecimento gerado por pesquisadores de todas as áreas (COSTA \& LEITE, 2006).

Como o Ipea produz conhecimento técnico (que serve para subsidiar ações governamentais) e científico (que visa a contribuir para o debate científico, em especial na área de economia) - os pesquisadores do Ipea, inclusive, publicam trabalhos em revistas e participam de eventos científicos -, o RCIpea tem características tanto de repositório do conheci- mento (descrito na literatura de GC) quanto de repositório institucional (citado na literatura sobre comunicação científica).

Nesse sentido, o repositório maximiza e acelera o impacto das pesquisas e, consequentemente, sua produtividade, progresso e recompensas (BRODY \& HARNAD, 2004). Resultados de pesquisas recentes apontam para isso. Lawrence (2001), por exemplo, apresenta resultados de um estudo que mostrou um crescimento de $336 \%$, em média, nas citações a artigos disponíveis online, em relação a artigos publicados off-line, na mesma fonte. (COSTA \& LeITE, 2006).

Tendo em vista essa abordagem, tanto como prática quanto como estratégia, para maximizar a visibilidade da pesquisa, os repositórios institucionais são hoje, no contexto brasileiro, uma realidade.

O RCIpea disponibiliza a produção do Ipea segundo os princípios da Iniciativa de Arquivos Abertos, ou Open Archives Initiative (OAI). Além da sua produção, o RCIpea disponibilizará no futuro também a produção técnica e científica de instituições parceiras, que desenvolvem e produzem trabalhos relevantes no campo de atuação do Ipea, nas diversas áreas relacionadas ao Desenvolvimento Econômico e Social. Para tanto, tais instituições também devem seguir as especificações do OAI.

A Iniciativa de Arquivos Abertos teve início em 1999, quando Ginspard, Luce e Van de Sompel fizeram uma chamada para explorar a cooperação entre arquivos de e-prints. Isso foi possível devido aos ideais e conceitos estabelecidos pela OAI e resumidos como: uso do software livre, também chamado Open Source; criação de repositórios de acesso aberto (Open Access); uso de padrões de preservação de objetos digitais etc. (Kuramoto, 2007). 
Nesse contexto, o termo archive significa repositório. O termo open é utilizado na perspectiva da arquitetura da informação, no sentido de definir e adotar interfaces de máquina que promovam a disponibilidade de conteúdos (Lagoze \& Van De Sompel, 2001).

Além disso, a OAI estabeleceu o Open Archives Initiative Protocol for Metadata Harvesting (OAI-PMH), um protocolo de comunicação que possibilita a coleta de metadados a partir de determinados provedores de dados. Esse protocolo, juntamente com um padrão de metadados, gera alto nível de interoperabilidade entre os repositórios. Os metadados também são apresentados em um padrão. O padrão de metadados mais utilizado é o Dublin Core (DC), porém, o OAI permite a adoção de outros padrões de metadados.

O Ipea, na implementação do seu repositório institucional, tendo em vista a interoperabilidade com outros repositórios, utilizou o Dspace, software Open Source; adotou o Dublin Core (DC) como padrão de metadados e habilitou o Open Archives Initiative Protocol for Metadata Harvesting (OAI-PMH).

\section{A construção do protótipo do} Repositório do Conhecimento do Ipea (RCIpea)

O protótipo do Repositório do Conhecimento do Ipea foi construído por um grupo de trabalho multidisciplinar. Participaram desse grupo representantes das seguintes áreas: Gestão do Conhecimento (CGPGO); Gestão de Projetos (DVPPI); Ciência da Informação (Biblioteca); Tecnologia da Informação e Comunicação (CGTIC); Assessoria de Comunicação (ASCOM) e ainda representantes das áreas finalísticas do Ipea, ou seja, técnicos de planejamento e pesquisa (pesquisadores).

O trabalho em grupo objetivou, além de buscar legitimidade para o projeto, a colaboração e a participação das áreas do instituto que se relacionariam futuramente com o repositório. O trabalho foi enriquecido pela equipe multidisciplinar, que representou a diversidade dos usuários do sistema de informação em construção.

\section{Definição das diretrizes para o desenvolvimento do acervo}

Nessa etapa buscaram-se definir as diretrizes para o desenvolvimento do acervo. Tais diretrizes visam a embasar o planejamento das coleções, oferecendo parâmetros de consistência e qualidade ao acervo e evitando o crescimento desordenado das coleções. Foram definidos os objetivos gerais do acervo e as estratégias de prospecção, seleção, alimentação e disponibilização dos objetos digitais, de acordo com: 1) os interesses e metas do Ipea; 2) os serviços de informação que seriam disponibilizados; e 3) o perfil dos usuários.

Instalação e customização do
software Dspace e aplicação da
identidade visual
Após a definição das diretrizes do
acervo, instalamos a versão mais atualizada
do software Dspace/MIT. Uma das diretrizes
definidas, na primeira etapa, foi manter o
software sem alterações significantes em seu
código-fonte. Dessa maneira, teríamos
mais facilidades na atualização das futuras
versões do Dspace, usufruindo dos aperfei-
çoamentos propostos pela comunidade
responsável pelo seu desenvolvimento e
manutenção. A principal alteração feita no
código foi justamente para conferir uma
identidade visual ao repositório.


Realização do estudo do domínio do repositório e definição da sua estrutura hierárquica (arquitetura informacional)

Segundo Brascher, os sistemas de organização do conhecimento (SOC) são sistemas conceituais que representam determinado domínio do conhecimento por meio da sistematização dos conceitos e das relações semânticas existentes entre eles. Englobam sistemas de classificação, cabeçalhos de assunto, arquivos de autoridade, tesauros, taxonomias e ontologias.

A organização da informação no Repositório do Conhecimento do Ipea (RCIpea) foi estabelecida a partir de sete eixos temáticos e dos grandes temas da área de Desenvolvimento Econômico e Social. Os eixos são utilizados pela instituição como macrolinhas de pesquisa. São eles: 1) inserção internacional soberana, 2) macroeconomia para o desenvolvimento, 3 ) fortalecimento do Estado, das instituições e da democracia, 4) estrutura tecnoprodutiva integrada e regionalmente articulada, 5) infraestrutura econômica, social e urbana, 6) proteção social, garantia dos direitos e geração de oportunidades, e 7) sustentabilidade ambiental. Já os grandes temas foram extraídos do estudo do domínio do repositório, por meio da análise de conceitos e das relações semânticas do MacroThesaurus da Organização para a Cooperação e Desenvolvimento Econômico (OCDE), chamado MacroThesaurus for Information Processing in the Field of Economic and Social Development (Quinta Edição).

Existem diferenças na organização da informação em função das ferramentas utilizadas. No Dspace, software utilizado para a construção do Repositório do Conhecimento do Ipea (RCIpea), a organização principal está estabelecida em comunidades, subcomunidades e coleções. As comunidades representam o $1^{\varrho}$ nível de organização da informação, as subcomunidades representam o $2^{\circ}$ nível de organização da informação, e as coleções representam o $3^{\circ}$ nível de organização da informação.

O Dspace também apresenta organizações secundárias, que permitem visualizar os documentos ou objetos digitais do repositório de diferentes maneiras. Considerou-se aqui a proposta conceitual de

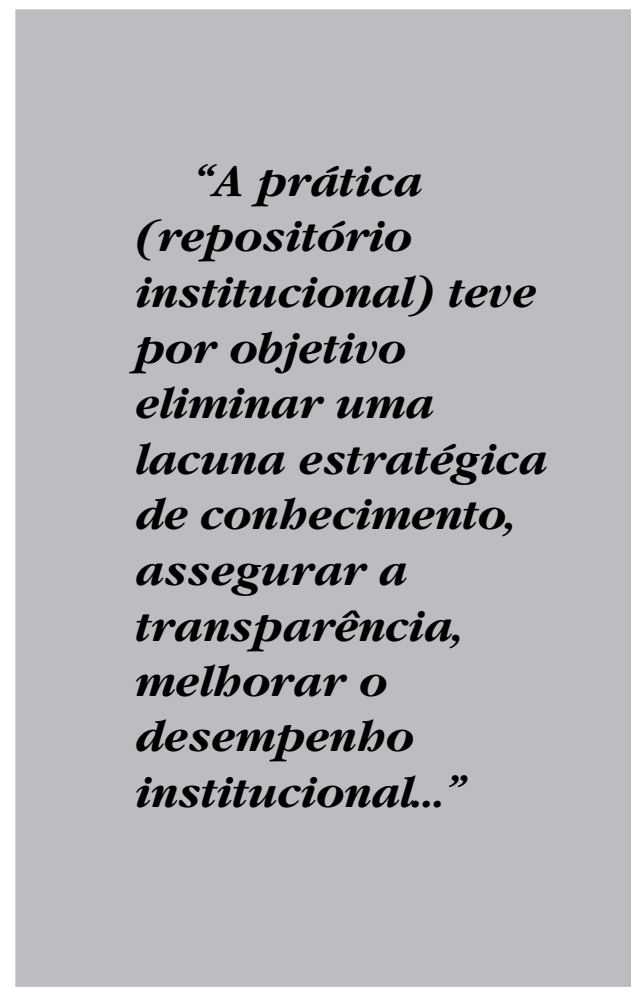

Brascher e Café, que definem a organização da informação (OI) mostrando os aspectos que a diferenciam da organização do conhecimento (OC). Segundo as autoras, a OI é um processo que envolve a descrição física e temática dos objetos informacionais. Ela compreende a organização de um conjunto de objetos, para arranjá-los sistematicamente em coleções, como a 
organização da informação em bibliotecas, museus, arquivos, tanto tradicionais quanto eletrônicos. A OI resulta na representação da informação, compreendida como o conjunto de atributos que representa determinado objeto informacional, obtido pelos processos de descrição física e de conteúdo (BRASCHER \& CAFÉ, 2008).

Essas organizações secundárias no repositório do Ipea são possíveis em função da descrição física e temática dos objetos informacionais digitais, que compreendem o preenchimento dos seus metadados. Como resultado desse tratamento informacional, no RCIpea é possível visualizar o acervo percorrendo listas de autores, títulos, assuntos e tipos de objeto.

As comunidades, que representam o $1^{\circ}$ nível de organização da informação, são os sete eixos temáticos para o desenvolvimento. As subcomunidades, que representam o $2^{\circ}$ nível de organização da informação, são os grandes temas da área de Desenvolvimento Econômico e Social, resultantes do estudo do domínio do repositório. Por fim, as coleções, que representam o $3^{\circ}$ nível de organização da informação, foram definidas a partir da tipologia documental do acervo.

Em relação ao $1^{\circ}$ nível de organização da informação, a escolha dos sete eixos temáticos para o desenvolvimento da organização da informação no RCIpea deu-se em função da importância deles para a instituição. Todos os estudos, pesquisas e demais atividades técnicas do Instituto de Pesquisa Econômica Aplicada (Ipea) são desenvolvidos em função dos sete eixos temáticos para o desenvolvimento.

O $1^{\circ}$ nível de organização da informação no RCIpea é, inicialmente, fixo, ou seja, não é extensível. Não será ampliado de acordo com as necessidades das diversas áreas, mas poderá ser modificado se os eixos temáticos para o desenvolvimento assim o forem.

As subcomunidades, que são o $2^{\circ}$ nível de organização da informação, são os grandes temas da área de Desenvolvimento Econômico e Social. A partir do estudo do domínio do RCIpea, foram identificados inicialmente 26 grandes temas. As 26 subcomunidades inicialmente definidas poderão, além de ser ampliadas, conter um terceiro ou quarto nível temático.

As subcomunidades são dinâmicas e flexíveis, ou seja, são extensíveis e adaptáveis. Poderão ser ampliadas ou alteradas, com base no estudo do domínio do RCIpea, de acordo com a necessidade de especificação dos assuntos das áreas técnicas da instituição.

\section{Definição do padrão de metadados e do formulário de entrada de dados}

O repositório foi construído segundo recomendações internacionais para disseminação da informação, visando a aumentar a visibilidade e o acesso da pesquisa, bem como a interoperabilidade de toda a produção do Ipea em rede.

O conjunto de metadados do Repositório do Conhecimento do Ipea (RCIpea) foi definido segundo o padrão Dublin Core. O padrão Dublin Core é um esquema de metadados que visa a descrever objetos digitais, tais como: vídeos, mapas, animações, softwares, sons, imagens, textos, sites na web etc.

A Dublin Core Metadata Initiative (DCMI) é uma organização dedicada a promover a adoção de padrões de interoperabilidade de metadados e desenvolver vocabulários especializados para descrever objetos digitais, que tornem 
mais inteligentes os sistemas de recuperação da informação.

A qualificação dos metadados foi necessária em função das necessidades do Ipea, e realizada conforme orientações da Dublin Core Metadata Initiative (DCMI). O DCMI estabeleceu maneiras padronizadas para qualificar ou refinar os elementos e encorajar o uso de esquemas de codificação e vocabulário. Cada metadado Dublin Core é opcional e pode ser repetitivo. Além disso, não há ordem no padrão Dublin Core para apresentar ou utilizar os metadados (elementos de dados).

O princípio orientador para a qualificação dos elementos Dublin Core afirma que uma aplicação que não compreende o metadado qualificado deve ser capaz de ignorar o qualificador e tratar o valor dos metadados, como se fosse um elemento absoluto. Embora isso possa resultar em alguma perda de especificidade, o valor dos elementos restantes (sem o qualificador) deve continuar a ser correto e útil para a recuperação da informação e para a interoperabilidade em rede.

O sistema é capaz de fazer a recuperação dos documentos pelo texto completo ou por meio de buscas simples e avançada. Não há limite em relação ao tamanho e formato dos arquivos. Além disso, estarão disponíveis informações estatísticas de acesso e downloads. Serão também mostradas no repositório as relações existentes entre as publicações do Ipea, em seus diversos suportes eletrônicos.

\section{Definição do fluxo geral do reposi- tório e do fluxo de submissão dos objetos digitais \\ O fluxo geral do repositório foi defi- nido conforme as necessidades iniciais do protótipo, que não contempla o autoarqui- vamento e a revisão midiático-pedagógica.}

Utilizamos uma funcionalidade do Dspace que permite somente desabilitar algumas etapas do fluxo, não excluindo definitivamente as possibilidades futuras de inclusão de novos atores no fluxo.

O fluxo de submissão dos objetos digitais foi organizado em sete passos, a saber: 1) perguntas iniciais: 1.1 Objeto publicado anteriormente em outra fonte? $1.2 \mathrm{O}$ objeto possui título alternativo? $1.3 \mathrm{O}$ objeto possui mais de um arquivo?; 2) indicação da coleção, que compreende a classificação do objeto na coleção e mostra a localização física principal do objeto no repositório; 3) preenchimento dos metadados do bloco de descrição geral; 4) preenchimento dos metadados do bloco de descrição temática; 5) preenchimento dos metadados do bloco de direitos autorais; 6) Upload e 7) publicação no RCIpea.

\section{Definição da política de acesso aberto e direitos autorais}

A política de acesso aberto e de direitos autorais do Repositório do Conhecimento do Ipea (RCIpea) visa a nortear a implementação do acesso aberto e a gestão dos direitos autorais no RCIpea, garantindo a não violação dos direitos autorais, de ordem patrimonial ou moral, resguardando todos os envolvidos de futuras implicações legais.

Pretende-se garantir dessa maneira o depósito, a disponibilização e o uso dos objetos digitais em conformidade com: 1) a legislação brasileira (Lei no 9.610/ 1998); 2) os tratados internacionais sobre o assunto; e 3) os objetivos do Instituto de Pesquisa Econômica Aplicada (Ipea).

Além de nortear a implementação do acesso aberto e a gestão dos direitos autorais no RCIpea, a política de acesso aberto e direitos autorais objetiva também: 1) estabelecer instrumentos de negociação 
padronizados para a negociação dos direitos autorais no Ipea; 2) especificar quais são as permissões e restrições de uso da produção técnica e científica do Ipea no RCIpea, em qualquer suporte ou formato eletrônico; 3) orientar, com base na Lei Brasileira de Direitos Autorais (Lei $\mathrm{n}^{\circ}$ 9.610 de 1998), como deve ser feita a citação e referência aos autores e colaboradores nos próprios objetos digitais; 4) estabelecer regras e critérios para o preenchimento dos instrumentos de negociação e dos metadados relacionados aos direitos autorais e conexos, como por exemplo: autoria (individual, coletiva, institucional, organizadores), colaborações que geram direitos autorais (tradução, dublagem, ilustração etc), outras colaborações, detentores de direitos autorais, permissões e restrições de uso, tipo ou regime de negociação, validade, necessidade de registro na Biblioteca Nacional etc.; 5) esclarecer conceitos e disponibilizar informações sobre diversos assuntos relacionados aos temas acesso aberto e direitos autorais, tais como direitos morais e patrimoniais do autor, direitos de imagem e conexos, sistema de licenciamento Creative Commons, licenças comuns, termos de cessão de direitos, cessão gratuita, cessão parcial, cessão não exclusiva, domínio público, plágio, violação de direitos autorais, exceções à Lei Brasileira de Direitos Autorais etc.

\section{Definição das regras de preen- chimento dos metadados}

Tão importante quanto definir os metadados, é definir as regras de preenchimento para cada um deles. Por essa razão, iniciamos os estudos que fundamentarão a definição dessas regras, que compreendem estudos relacionados à catalogação e classificação (descrição física e temática) de diferentes tipos de objetos digitais, garantindo assim o seu correto preenchimento e recuperação.

No RCIpea, foi definido apenas um formulário de entrada de dados para todos os tipos de objetos digitais. Foram definidos metadados obrigatórios, que são utilizados para descrever todos os tipos de objetos digitais, como, por exemplo, autor e título. Outros metadados são facultativos, pois alguns objetos digitais não requerem a utilização de todo o conjunto de metadados para a descrição física e temática. Nesse caso, somente os metadados não obrigatórios poderão não ser utilizados na descrição.

\section{Considerações finais}

Com a implementação do Repositório do Conhecimento do Ipea (RCIpea), parte do conhecimento tácito pode ser explicitado e, juntamente com os demais conhecimentos explícitos da instituição, pode hoje ser representado, organizado e armazenado de forma estruturada. Além disso, tal conhecimento pode ser disseminado com base em padrões internacionais de interoperabilidade, promovendo, assim, um acesso bem mais amplo dos públicos interno e externo à produção técnica e científica da organização.

Esse caso mostra a importância da adoção, por parte de organizações públicas como o Ipea, de um modelo de GC holístico (que considera os vários aspectos da GC: alinhamento estratégico, viabilizadores, ciclo de GC, ciclo KDCA, resultados e partes interessadas). Ilustra também a relevância de se utilizar um método de implementação voltado para eliminar lacunas estratégicas do conhecimento e que conduz a organização a definir uma visão de GC, objetivos, estratégia e 
plano de GC, assim como indicadores para verificar se os resultados da estratégia estão sendo alcançados.

Não se resume, como procuramos demonstrar, a apenas uma questão de implementar uma prática ou ferramenta isolada de GC. A prática (repositório institucional) teve por objetivo eliminar uma lacuna estratégica de conhecimento, assegurar a transparência, melhorar o desempenho institucional e contribuir para o cumprimento da missão do Ipea de produzir, articular e disseminar conhecimento para aperfeiçoar políticas públicas e contribuir para o planejamento do desenvolvimento brasileiro. Além disso, é importante destacar que a prática está alinhada com o modelo de GC e com o método de implementação.

(Artigo recebido em março de 2013. Versão final em abril de 2013).

\section{Referências bibliográficas}

Alvarenga Neto, R. C. D. de; Vieira, J. L. G. V. Building a knowledge management (KM) model at Brazil's Embrapa (Brazilian Agricultural Research Corporation): towards a knowledge-based view of organizations. Electronic Journal of Knowledge management, v. 9, Issue 2, 2011.

Apo. Knowledge management. facilitator's Guide, 2009. Disponível em: http://www.apotokyo.org/00e-books/IS-39_APO-KM-FG.htm. Acessado em: 9 dez. 2011.

BAтіsтA, Fábio Ferreira. Modelo de gestão do conhecimento para a administração pública brasileira: como implementar a gestão do conhecimento para produzir resultados em benefício do cidadão. Brasília, DF: Instituto de Pesquisa Econômica Aplicada (Ipea), 2012. Disponível em: http://repositorio.ipea.gov.br/handle/11058/754. Acesso em 28 fev. 2013.

B APTISTA, A. A. et al. Comunicação cientifica: o papel da Open Archives Initiative no contexto do Acesso Livre. Disponível em: http://journal.ufsc.br/index.php/ eb/article/view/ 1518-2924.2007v12nesp1p1/435 Acesso em: 23 fev. 2013.

BARBOSA, J. G. P. et al. A proposed architecture for implementing a knowledge management system in the Brazilian National Cancer Institute. Brazilian administration Review, v. 6, n. 3, p. 247-262, July/Sept. 2009. Disponível em: http://www. anpad. org. br/periodicos/ arq_pdf/a_888.pdf Acesso em: 11 nov. 2011.

Brascher, M.; CAFÉ, L. Organização da Informação ou Organização do Conhecimento? In: IX ENANCIB. ANCIB, 2008. Disponível em: http://skat.ihmc.us / rid=1KR7TM7S9-S3HDKP-5STP/BRASCHER \%20CAF\%C3\%89 (2008)-1835.pdf Acesso em: 23 fev.2013.

Brody, Tim; Harnad, Stevan. The research impact cycle. Disponível em: http:// docs.lib.purdue.edu/context/iatul/article/1729/type/native/viewcontent Acesso em: 22 fev. 2013.

Costa, S. M. S; Leite, F. C. L. Repositórios institucionais como ferramentas de gestão do conhecimento científico no ambiente acadêmico. Perspectivas em Ciência da Informação, 
Belo Horizonte, v. 11 n. 2, p. 206-219, maio/ago. 2006. Disponível em: http:// www.scielo.br/pdf/\%0D/pci/v11n2/v11n2a05.pdf Acesso em: 22 fev. 2013.

DALKIR, Kimiz. Knowledge management in Theory and practice. 2. ed.. Cambridge: Massachusetts Institute of Technology, 2011.

European Km Forum. Standardized KM implementation. 2001. Disponível em: http:// www.providersedge.com/docs/km_articles/Standardised_KM_Implementation.pdf. Acessado em: 29 maio 2013.

FonseCA, A. Organizational Knowledge assessment methodology. Washington, D.C.: World Bank Institute, 2006.

Heisig, P. Harmonization of knowledge management - comparing $160 \mathrm{KM}$ frameworks around the globe. Journal of Knowledge Management, v. 13, n. 4, p. 4-31, 2009.

Instrumento para a Avaliação da Gestão Pública. Ciclo 2010. Disponível em: http:/ /www.gespublica.gov.br/projetos-acoes/pasta.2010-04-26.8934 490474/ Instrumento_ciclo_2010_22mar.pdf Acessado em: 10 de dez. 2011.

Lagoze, C.; Sompel, H. V. de. The Santa Fe Convention of the Open Archives Initiative. Dlib Magazine, vol. 6. n. 2, february 2000. Disponível em: http://www.dlib .org/dlib/ february00/vandesompel-oai/02vandesompel-oai.html Acesso em: 22 fev. 2013.

LAWRENCE, Steve. Free online availability substantially increases a paper's impact. Nature webdebates. Disponível em: http://www.nature.com/nature/debates/e-access/Articles/ lawrence.html?ref=Penisbuyutucuu.com Acesso em: 22 fev. 2013.

Medeiros, G.M. Organização da informação em repositórios digitais. UFSC, 2012. Disponível em: http://repositorio.ufsc.br/handle/123456789/9165. Acesso em: 05 maio 2012.

Nonaka, I.; TAkeuchi, H. The knowledge-Creating Company. How Japanese companies create the dynamics of innovation. New York: Oxford University Press, 1995.

OECD. Survey of knowledge management practices in ministries/ departments/agencies of central government. Paris: Oecd, 2003 [Fontes: Documentos Oecd: Gov/Puma (2003) 17 mar. 2003; Puma/HRM (2002) 23 jan. 2002; Puma/HRM (2001) 8 jun. 2001].

Salavati; A.; Shafei, R.; Shaghayegh, E. A model for adoption of knowledge management in Iranian public organizations. European Journal of Social Sciences, v. 17, n. 1, 2010.

THE KNOW NeTWORK. Oito critérios mundiais na certificação internacional de auditores em processos de gestão do conbecimento - Metodologia MAKE - Most Admired Knowledge Enterprises. The Know Network. Disponível em: http://www.premiomake.com.br/uploads/6/1/ 1/8/6118855/folder-curso_make_janeiro-fevereiro_2012-sao_paulo.pdf. Acesso em: 29 maio 2013. 


\title{
Resumo - Resumen - Abstract
}

\begin{abstract}
Alinhando o modelo, o método de implementação e a prática de gestão do conhecimento (GC): o caso do Repositório do Conhecimento do Instituto de Pesquisa Econômica Aplicada (RCIpea)
\end{abstract}

Fábio Ferreira Batista e Veruska da Silva Costa

O objetivo deste artigo é analisar como o Instituto de Pesquisa Econômica Aplicada (Ipea) implementou de forma alinhada um modelo, um método e uma prática de gestão do conhecimento (GC) (repositório do conhecimento). O referencial teórico utilizado é o Modelo de Gestão do Conhecimento para a Administração Pública Brasileira construído por um dos autores deste trabalho após a realização de uma revisão sistemática da literatura sobre modelos de GC na administração pública (BATISTA, 2012). O trabalho iniciou-se com a escolha do modelo. Em seguida, utilizou-se de um roteiro para elaborar o plano de GC. O foco desse plano foi a implementação do repositório institucional. As principais conclusões deste artigo são: i) como resultado da implementação do repositório, parte do conhecimento tácito pode ser explicitado (na forma de narrativas, por exemplo) e, juntamente com os demais conhecimentos explícitos, pode hoje ser representado, organizado e armazenado de forma estruturada; ii) o Ipea implementou não apenas uma prática isolada de GC, mas conseguiu alinhar a prática (repositório) com um modelo e método de implementação de GC; e iii) a importância da adoção, por parte de organizações públicas como o Ipea, de um modelo de GC holístico, isto é, com uma visão integral de GC.

Palavras-chave: Modelo de Gestão do Conhecimento; método de implementação de gestão do conhecimento; repositório institucional de acesso aberto

\section{Alineando el modelo, el método de implementación y la práctica de gestión del conocimiento (GC): el caso del Repositorio del Conocimiento del Instituto de Pesquisa Econômica Aplicada (RCIpea) \\ Fábio Ferreira Batista y Veruska da Silva Costa}

El propósito de este artículo es analizar cómo el Instituto de Investigação Econômica Aplicada (Ipea) puso em práctica un modelo, un método y una práctica de GC de forma integrada. El marco teórico de este trabajo es el Modelo de Gestión del Conocimiento para la Administración Pública Brasileña construido por uno de los autores de este trabajo tras llevar a cabo una revisión sistemática de la literatura sobre modelos de GC en la administración pública (BATISTA, 2012). El trabajo tuvo inicio tras la elección del modelo: Modelo de Gestión del Conocimiento para la Administración Pública Brasileña. En seguida, se formuló un guión para elaborar el Plan de GC. El enfoque del plan fue la implementación del repositorio con vistas a eliminar una brecha de conocimiento. Las principales conclusiones de este artículo son: i) como resultado de la implementación del repositorio, parte del conocimiento tácito puede ser explicitado (en la forma de narraciones, por ejemplo) e, junto con los otros conocimientos explícitos, ahora se puede representar, organizar y almacenar de forma estructurada; ii) IPEA puso en ejecución no sólo una práctica aislada GC, pero ha logrado integrar la práctica (repositorio) con un modelo y método de aplicación GC; e iii) la importancia de la adopción -por organizaciones públicas como el IPEA- de um modelo de GC holístico, es decir, con una visión integral de la GC.

Palabras clave: Modelo de Gestión del Conocimiento; Método de Implementación de Gestión del Conocimiento; Repositorio Institucional de Acceso Abierto 
Matching the model, the method of implementation and the practice of knowledge management: the case of the Institutional Repository of the Institute for Applied Economic Research (Ipea) in Brazil

Fábio Ferreira Batista and Veruska da Silva Costa

This paper aims to assess how the Institute for Applied Economic Research (Ipea) was able to implement a KM framework, a KM method and a KM practice in an integrated way. The theory behind this work is the KM Framework for the Brazilian Public Administration designed by one of the authors of this paper after a literature review about KM frameworks in the public administration (BATISTA, 2012). Following this decision, a KM Plan was draft based on an implementation method designed for that purpose. The plan main focus was the implementation of a repository to eliminate a knowledge gap and to improve organizational performance. This article main conclusions are: i) as a result of the repository implementation, part of Ipea's tacit knowledge (for example in the form of storytelling) can be made explicit and, together with other explicit knowledge, can now be represented, organized and stored in a structured form; Ipea did not implemented a single KM practice, but it was able to integrate the practice (repository) with a KM framework and KM implementation method; and iii) the importance of the adoption of a holistic (or systematic view) KM framework by public organizations, such as Ipea.

Keywords: Knowledge Management Framework; KM Implementation Method; Open Access Institutional Repository

Fábio Ferreira Batista

Doutor em Ciência da Informação. Técnico de Planejamento e Pesquisa do Instituto de Pesquisa Econômica Aplicada (Ipea). Professor da Universidade Católica de Brasília (UCB). Contato: fabio.batista@ipea.gov.br

Veruska da Silva Costa

Bacharel em Biblioteconomia. Gerente do Projeto Repositório do Conhecimento do Ipea (RCIpea), no Instituto de Pesquisa Econômica Aplicada (Ipea). Contato: veruska.costa@ipea.gov.br 